\title{
Role of CT in Assessment and Characterization of Renal Masses
}

\author{
Satish Patil ${ }^{1}$, Shivanand Patil ${ }^{2}$, Vishal Nimbal ${ }^{3}$ \\ ${ }^{1}$ Assistant Professor, ${ }^{2}$ Assistant Professor, ${ }^{3}$ Senior Resident, Shri B M Patil Medical College Hospital and Research Centre, \\ Bijapur, Karnataka, India
}

Corresponding author: Dr. Shivanand Patil, Assistant professor, Shri B M Patil Medical College Hospital and Research Centre, Bijapur, Karnataka, India

DOI: $10.21276 / \mathrm{ijcmsr} .2018 .3 .2 .41$

How to cite this article: Satish Patil, Shivanand Patil, Vishal Nimbal. Role of CT in assessment and characterization of renal masses. International Journal of Contemporary Medicine Surgery and Radiology. 2018;3(2):B174-B179.

\section{A B S T R A C T}

Background: The purpose of our study is to compare thin-section corticomedullary and nephrographic phase images of the kidneys to determine whether one of these phases of parenchymal enhancement is superior in the characterization of a previously detected indeterminate renal mass.

Materials and Methods: This was a cross sectional prospective study which included 30 consecutive cases of renal masses detected on MDCT. The study data was collected from July 2014 to June 2016 in the Department of Radio-diagnosis, Shri B.M. Patil Medical College Hospital and research center, Bijapur, Karnataka. Attenuation values and enhancement pattern of renal masses during unenhanced, corticomedullary and nephrographic phases were analysed, for better detection and characterization of renal masses by multidetector computed tomography.

Results: No statistically significant differences $(p>0.05)$ in enhancement were noted for the radiologically benign cysts when the corticomedullary and nephrographic phases were compared. The normal renal cortex demonstrated greater

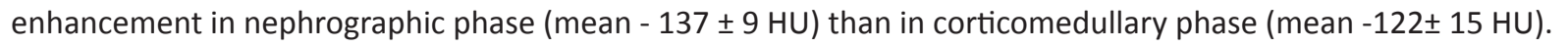

Conclusion: To conclude, MDCT protocol for evaluation of renal masses should include unenhanced, corticomedullary and nephrographic phases for better detection and characterization of renal masses.

Keywords: Nephrographic, Corticomedullary Phases, Characterization of Renal Masses.

\section{INTRODUCTION}

The most recent technical advances, obtained with the use of multidetector CT (MDCT) and with multiple reconstruction software, allow a significant reduction of the scanning time associated with increased spatial resolution. The reduced gantry rotation times ( $0.5 \mathrm{~s}$ or less) allow about 25 times faster scanning times than traditional single row helical CT. Faster scan times results in decreased breath-hold times with reduction of motion artifacts. The use of thinner slice thickness is associated to better quality volume data set for workstation analysis and multiplanar reformation (MPR): thus, three dimensional (3-D) imaging and volume reconstructions are possible, with more and improved diagnostic images. The main advantages of MDCT are faster scanning time, increased volume coverage and improved spatial and temporal resolution. ${ }^{1}$

Renal cell carcinoma (RCC) is the most common adult renal epithelial cancer, accounting for more than $90 \%$ of all renal malignancies. ${ }^{2} \mathrm{RCC}$ is the most lethal of all urologic cancers. The 5-year cancer-specific survivals of patients with pT4 RCC and lymph node metastases are 20\% and 5\%$30 \%$, respectively. There is continued global increase in the incidence of RCC, partly due to early diagnosis with cross sectional imaging modalities.

The great majority of renal masses are found incidentally on imaging most of these benign some are malignant and need to be surgically removed.

Therefore, the proper characterization of these masses is essential so that appropriate management is instituted.

\section{Objective of the study}

- To study attenuation and enhancement pattern of renal masses during unenhanced, corticomedullary and nephrographic phases.

- To evaluate the characteristics of renal parenchymal enhancement during corticomedullary and nephrographic phases.

- To compare computed tomography finding with pathological diagnosis, Where ever possible.

\section{MATERIAL AND METHODS}

This was a cross sectional prospective study which included 30 consecutive cases of renal masses detected on MDCT. The study data was collected from July 2014 to June 2016 in the Department of Radio-diagnosis, Shri B.M. Patil Medical College Hospital and research center, Bijapur, Karnataka. Attenuation values and enhancement pattern of renal masses during unenhanced, corticomedullary and nephrographic phases were analysed, for better detection and characterization of renal masses by multidetector computed tomography.

Patients with suspected (hematuria, flank pain, flank fullness) or incidentally /utrasonographically detected renal 
mass referred for $\mathrm{CT}$ scan of abdomen to the Department of Radiodiagnosis, Shri B.M. Patil Medical College Hospital and research center, Bijapur. Patients with renal masses on CT imaging were included in this study.

\section{Inclusion criteria:}

All patients with renal mass on $\mathrm{CT}$ abdomen were included irrespective of age and sex.

\section{Exclusion Criteria}

- Simple renal cyst (Bosniak category 1) - confirmed on ultrasonography.

- Renal trauma.

- Extra renal mass invading renal parenchyma.

All solid lesions with attenuation similar to other soft tissue abdominal structures and if interrogation of region of interest revealed tissue enhancement of $10 \mathrm{HU}$ or greater were classified as malignant.

For characterization of cystic renal lesions, the Bosniak criteria were used. ${ }^{3}$

\section{STATISTICAL ANALYSIS}

The statistical analysis was done using IBM SPSS version 20. The descriptive data for gender, enhancement characteristics, location and distribution of lesions was calculated. Chi square test was used to assess the association between subtype of renal masses (benign or malignant) and gender, morphological features, and type of contrast enhancement. To assess the association between benign and malignant masses with respect to age, size of lesion, contrast enhancement in corticomedullary and nephrographic phases' student $T$ test was used. The diagnostic efficacy and cut off values of enhancement and degree of enhancement in various phases was determined by receiver operating characteristic (ROC) curve. The curves were analyzed for cut off values to differentiate RCC from other masses. In all our analysis $\mathrm{p}$ value $<0.05$ was significant.

\section{RESULTS}

The present study is a cross sectional study including 30 patients who had renal masses which was detected on MDCT between July 2014 - June 2016.

In this series, the mean age of patients was $53 \pm 12$ years (range 26 to 82 years) which include 19 males and 11 females (table-1,2).

The mean size of renal masses was $5.463 \pm 3.5744$ (range 2 to $18 \mathrm{~cm})($ table- 3,4$)$.

\section{Radiological features of benign and malignant lesions}

a. Enhancement pattern in benign and malignant lesions The enhancement pattern among benign renal masses was homogenous in 7 cases and heterogenous in 7. Among malignant masses 13 out of 15 cases enhanced heterogenously and only 2 cases were homogenous. The variability in enhancement pattern between benign and malignant lesion was significant with $\mathrm{p}$ value $<0.05$.

\section{b. Tumor margin in benign and malignant lesions}

The benign masses were well defined in 12 out of 14 cases and ill-defined in 2 . The malignant masses were well defined in 7 out of 15 cases and ill-defined in 8 . The association

\begin{tabular}{|l|c|}
\hline Renal masses & Age in years \\
\hline Mean & 53.40 \\
\hline Std. Deviation & 12.746 \\
\hline Minimum & 26 \\
\hline Maximum & 82 \\
\hline \multicolumn{2}{|c|}{ Table-1: Age Distribution of Renal Masses } \\
\hline
\end{tabular}

\begin{tabular}{|l|c|c|}
\hline Sex distribution in renal masses & Frequency & Percent \\
\hline Male & 19 & 63.3 \\
\hline Female & 11 & 36.7 \\
\hline \multicolumn{2}{|c|}{ Table-2: Sex Distribution of Renal Masses } \\
\hline
\end{tabular}

\begin{tabular}{|l|c|}
\hline Renal masses & Size in cm \\
\hline Mean & 5.463 \\
\hline Std. Deviation & 3.5744 \\
\hline Minimum & 2.0 \\
\hline Maximum & 18.0 \\
\hline \multicolumn{2}{|c|}{ Table-3: Size of Renal Masses } \\
\hline
\end{tabular}

\begin{tabular}{|l|c|c|}
\hline & Frequency & Percent \\
\hline Right & 13 & 43.3 \\
\hline Left & 16 & 53.3 \\
\hline Bilateral & 1 & 3.3 \\
\hline \multicolumn{2}{|c|}{ Table-4: laterality of renal masses } \\
\hline
\end{tabular}

\begin{tabular}{|l|l|l|}
\hline $\begin{array}{l}\text { No of } \\
\text { cases }\end{array}$ & CT diagnosis & Pathological diagnosis \\
\hline 11 & RCC & RCC \\
\hline 2 & Bosniak type IV & RCC \\
\hline 1 & RCC & $\begin{array}{l}\text { Renal metastasis - } \\
\text { unknown primary }\end{array}$ \\
\hline 1 & Transitional cell carcinoma & $\begin{array}{l}\text { Transitional cell carci- } \\
\text { noma }\end{array}$ \\
\hline 7 & Renal abscess & Renal abscess \\
\hline 4 & AML & AML \\
\hline 2 & Bosniak type II Cyst & - \\
\hline 1 & Bosniak type III Cyst & Lost in follow up \\
\hline 1 & Oncocytoma & Oncocytoma \\
\hline \multicolumn{2}{|c|}{ Table-5: Histopathological correlation } \\
\hline
\end{tabular}

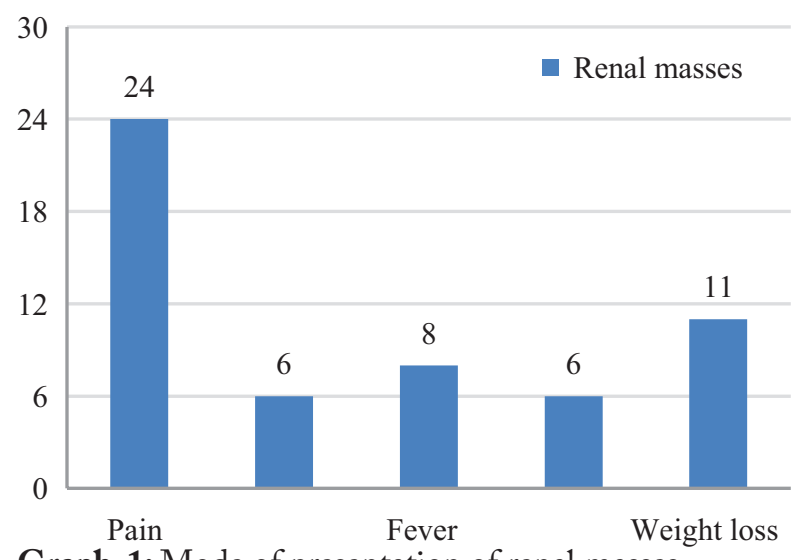

Graph-1: Mode of presentation of renal masses

between tumour margin and benign/malignant mass was significant $\mathrm{p}<0.05$ 


\begin{tabular}{|c|c|c|c|}
\hline & Benign & Malignant & $P$ value \\
\hline No of Cases & 14 & 15 & \\
\hline Mea Age (in years) & $45.71 \pm 11.605$ & $61.13 \pm 9.125$ & 0.001 \\
\hline Male to female ratio & $1: 1$ & $2.7: 1$ & 0.196 \\
\hline Size in $\mathrm{cm}$ & 5.6 & 5.56 & 0.981 \\
\hline \multicolumn{4}{|l|}{ Symptoms Present } \\
\hline Pain & 11 & 13 & 0.564 \\
\hline Hematuria & 1 & 5 & 0.82 \\
\hline Weight loss & 1 & 9 & 0.003 \\
\hline Lump & 2 & 4 & 0.411 \\
\hline Fever & 6 & 2 & 0.075 \\
\hline Well-defined/III-defined & $12 / 2$ & $7 / 8$ & 0.027 \\
\hline \multicolumn{4}{|l|}{ Attenuation } \\
\hline Hypodense & 13 & 13 & \\
\hline Isodense & 1 & 1 & \\
\hline Hyperdense & 0 & 1 & \\
\hline Enhancement Pattern & & & 0.033 \\
\hline Homogenous & 7 & 2 & \\
\hline Heterogenous & 7 & 13 & \\
\hline \multicolumn{4}{|l|}{ Hounsfield Units (HU) } \\
\hline Unenhanced phase & $9.29 \pm 21.514$ & $34.87 \pm 3.543$ & 0.001 \\
\hline Corticomedullary phase & $14.21 \pm 26.762$ & $96.53 \pm 12.977$ & 0.001 \\
\hline Nephrographic phase & $16.14 \pm 27.840$ & $72.93 \pm 10.194$ & 0.000 \\
\hline Degree of enhancement in Corticomedullary phase & $4.9286 \pm 11.40489$ & $61.6667 \pm 15.29550$ & \\
\hline Degree of enhancement in nephrographic phase & $6.8571 \pm 14.21190$ & $38.0667 \pm 10.85927$ & \\
\hline
\end{tabular}

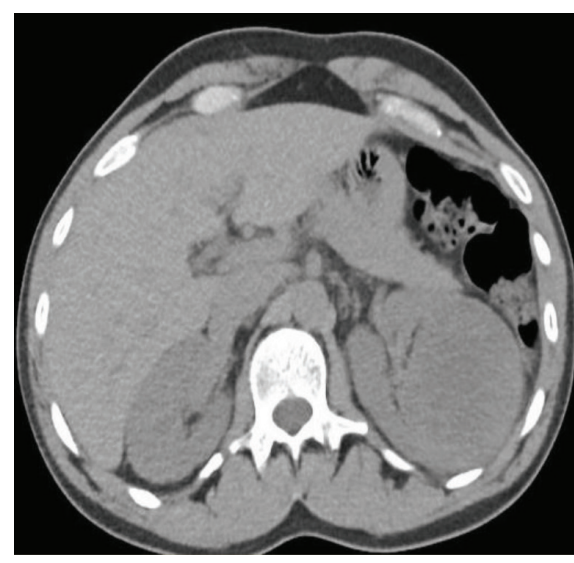

Figure-1: Shows the unenhanced phase

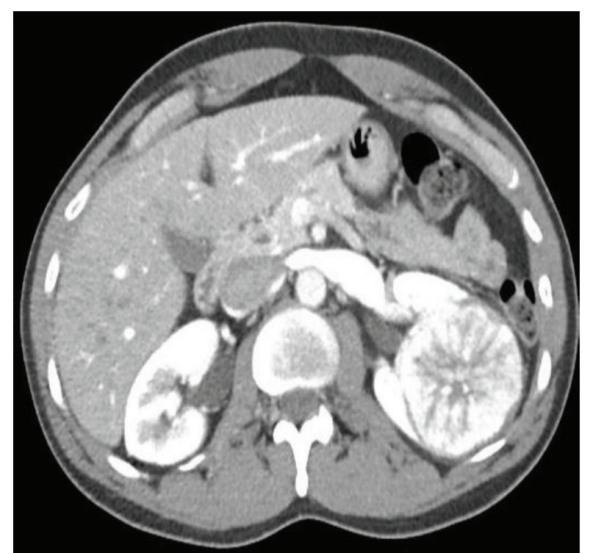

Figure-2: Shows the corticomedullary phase

c. Calcification in Simple renal cyst (Bosniak category 1) - confirmed benign and malignant lesions

Peripheral calcification was seen in 4 out of 14 cases among

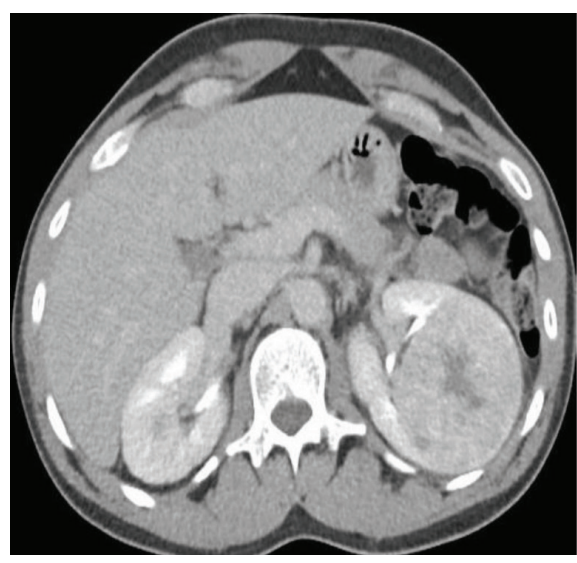

Figure-3: Shows the nephrographic phase

benign masses whereas malignant lesion did not show evidence of calcification.

\section{d. Density in benign and malignant lesions}

13 out of 14 benign cases presented as hypodense masses and one was isodense to renal cortex. 13 out of 15 malignant masses presented as hypodense masses and one cases each presented as isodense and hyperdense masses.

\section{Attenuation in Pre and Post Contrast Ct Images in Benign and Malignant Lesions}

The HU of renal masses were compared between benign and malignant masses in unenhanced and enhanced phases. In unenhanced sequences the benign masses showed a mean $\mathrm{HU}$ value of 9.29, the malignant masses showed a mean $\mathrm{HU}$ value of 34.87. In corticomeduary and nephrographic phases the mean HU in benign masses was 14.21 and 16.14 respectively. The malignant masses displayed rapid enhancement and washout in corticomedulary and nephrographic phases 
with a mean HU value of 96.53 and 72.93 respectively. The malignant masses showed greater mean enhancement in corticomedullary phase than in nephrographic phase. In contrary the benign masses - oncocytoma showed greater enhancement in nephrographic phase than in corticomedullary phase. The mean enhancement in different phases of enhancement and unenhanced phases between benign and malignant masses were statistically significant with $\mathrm{p}$ value $<0.05$.

\section{Age distribution and enhancement of Benign cystic lesions}

Two radiologically benign cysts of Bosniak type 2 were diagnosed. The mean age in cases with benign cysts was $46 \pm$ 16.9 years (range - 34 to 58 years). Cysts demonstrated mean Attenuation of $15 \pm 1.4 \mathrm{HU}$. The mean cyst enhancement was $1.5 \pm 3.5 \mathrm{HU}$ during Corticomedulary phase and $2 \pm 2.8$ HU during nephrographic phase. The differences in mean enhancement in corticomedullary phase and nephrographic phase was statistically insignificant.

\section{Age and size distribution and enhancement of angiomyolipoma}

In our study 4 cases of angiomyolipoma were included. All cases of angiomyoliopa were present in females. The mean age was $39.25 \pm 5$ years (range 35 to 45 years). The mean size was $9.3 \pm 7 \mathrm{~cm}(3 \mathrm{~cm}$ to $18 \mathrm{~cm})$. The masses showed a mean attenuation of $-22.75 \pm 1.5 \mathrm{HU}$ on unenhanced CT images. Cortical mean enhancement was $-19 \pm 3 \mathrm{HU}$ during corticomeduallary phase and $16.5 \pm 3.1 \mathrm{HU}$ during nephrographic phase.

\section{Age and size distribution and enhancement of renal abscess}

In our study 7 cases of renal abscess were included since most of the lesions mimicked as a mass. Out of 7 cases 3 were present in females and 4 in males. The mean age was $47.8 \pm 13$ years (range 26 to 63 years). The mean size was $3.9 \pm 1.8 \mathrm{~cm}$ $(2.1 \mathrm{~cm}$ to $7.2 \mathrm{~cm})$. The masses showed a mean attenuation of $22.4 \pm 1.7 \mathrm{HU}$ on unenhanced CT images. Cortical mean enhancement was $23 \pm 1.7 \mathrm{HU}$ during corticomeduallary phase and 24.14 $\pm 1.4 \mathrm{HU}$ during nephrographic phase. Pain was most common complaint seen in all cases and in 6 out of 7 cases (graph-1). The urine culture was positive in 6 out of 7 cases. Other features such as focal or global enlargement of the kidney, perinephric stranding, and thickening of Gerota fascia was seen in most cases. Based on these imaging findings the diagnosis of renal abscess was made. All the cases showed significant improvement after giving antibiotics \pm percutaneous drainage.

\section{TCC}

A case of transitional cell carcinoma was included in our study. It was seen in a 70 year old male patient who presented with weight loss. It was an ill-defined hypodense mass in pelvis extending to cortex and ureter. The attenuation on unenhanced CT images was $39 \mathrm{HU}$. On corticomedullary phase and nephrographic phase the attenuation was 82 and $76 \mathrm{HU}$ respectievely. On biopsy mass was found to be TCC.

\section{Oncocytoma}

A single case of oncocytoma was included in our study. It was seen a 56 year old male patient. It presented as a welldefined heterogenous mass on post contrast study. The attenuation of the mass on unenhanced CT images was 34 HU. On corticomedullary phase and nephrographic phase the attenuation was 78 and $89 \mathrm{HU}$ respectively. Unlike RCC and TCC, oncocytoma showed gradual enhancement in nephrographic phase compared to corticomedullary phase. RCC and TCC showed wash out in nephrographic phase. A characteristic central scar was noted in mass which helped in radiological diagnosis of oncocytoma and this was proven on biopsy also (figure-1,2,3).

A total of 13 cases of renal cell carcinoma was included in our study. Two cases of RCC presented as a solid cystic mass with enhancing soft tissue components with no evidence of metastasis or renal vessel involvement. These masses were diagnosed as Bosniak type 4 cysts based on imaging findings. The lesions turned out to be renal cell carcinoma on biopsy. One mass was diagnosed as renal cell carcinoma based on enhancement $>20 \mathrm{HU}$ on corticomedullary and nephrographic phases. On biopsy the mass lesion was diagnosed as metastasis (adenocarcinoma) from an unknown primary (table-5,6).

In one patient two mass lesions were detected in lower pole of right kidney. It was a well-defined cystic lesion with thick enhancing septations with no enhancing soft tissue component and it was classified as Bosniak type III cyst, however the patient refused biopsy and was lost in follow up and was excluded from the study.

Two cases of Bosniak type II cysts did not undergo any biopsy/surgical intervention because of its benign appearance on imaging.

\section{DISCUSSION}

MDCT continues to have a large impact on the diagnosis and characterization of renal masses. It is the only imaging modality which is required prior surgery. Advances in data acquisition and display provide tremendous capabilities in detection and management of renal masses.

Renal parenchymal tumours are heterogenous group consisting of benign to highly aggressive/malignant masses. The morphological features and the degree of enhancement vary significantly depending upon the type of tumour. The precise preoperative prediction of the histological type of lesion may be helpful not only for determining the appropriate treatment plan, such as the extent of the preoperative evaluation and surgery, but also in counselling the patient preoperatively. ${ }^{4}$

Out of 30 cases, 14 cases were benign and 15 were malignant masses. In one patient two mass lesions were detected in lower pole of right kidney. It was a well-defined cystic lesion with thick enhancing septations and it was classified as Bosniak type III cyst, however the patient refused biopsy and was lost in follow up and was excluded from the study. The benign lesions included 6 cases of renal abscess, 4 angiomylipoma, one oncocytoma and 2 cases of Bosniak type II cyst.

Among malignant masses, 13 cases were renal cell carcinoma. One transitional cell carcinoma of renal pelvis and one metastasis (adenocarcinoma) from unknown primary. RCC 
was the most common renal mass detected comprising of $43 \%$ cases of renal masses.

In a study by Birnbaum et al. ${ }^{5}$, in his series the average size of the neoplasms was $4.3 \mathrm{~cm} \pm 1.8$ (range, $1.4-8.0 \mathrm{~cm}$ ). The results of this study was comparable to our study.

In a study by CM Shetty et al. ${ }^{6}$ twenty three lesions were detected in twenty two patients. The size of the lesion ranged from 2.4-14 cm with mean size of $9.7 \mathrm{~cm}$.

The study conducted by Welch et al. ${ }^{7}$, size ranged from 1.5 to $19 \mathrm{~cm}($ mean $7 \mathrm{~cm}$ ). The mean size of renal masses in present study is slightly lower that the study conducted by $\mathrm{CM}$ Shetty et al. ${ }^{6}$ and Welch et al. ${ }^{7}$

In our study peripheral calcification was seen in 4 out of 14 cases among benign masses whereas malignant lesion did not show evidence of calcification. The calcification was noted in septae and walls of cystic masses.

Similarly in studies by Philip J. Weyman et al..$^{8}$ and Israel GM et al. ${ }^{9}$ peripheral calcifications in septae and walls was noted in benign cystic lesions.

\section{Comparison of enhancement of normal renal cortex.}

The renal cortex demonstrated a mean attenuation of $32 \pm 3$ $\mathrm{HU}$ on unenhanced CT images. Cortical mean enhancement was $122 \pm 15 \mathrm{HU}$ during corticomeduallary phase and $137 \pm 9$ HU during nephrographic phase. The degree of enhancement in corticomedullary and nephrographic phases were $90 \pm 16$ and $105 \pm 10$. There was a statically significant difference in enhancement in corticomedullary and nephrographic phase $(\mathrm{P}<0.05)$.

Similar results were noted in a study by $\mathrm{Cm}$ Shetty et al. ${ }^{6}$ Renal cortex showed greater enhancement in the nephrographic phase compared with that in the corticomedullary phase.

In studies by Cohan et al. ${ }^{10}$ and Szolar et al. ${ }^{11}$ the mean cortical enhancement was greater in corticomedullary than in nephrographic phases.

The discrepancy in the cortical enhancement between present study and Cohan et al. ${ }^{10}$ and Szolar et al. ${ }^{11}$ studies are due to substantial differences in the rate of contrast injection and due to differences in the time of acquisition.

In present study, corticomedullary and nephrographic phase images are acquired between $30-40$ seconds and $80-120$ seconds after initiation of contrast injection respectively. $100 \mathrm{ml}$ of contrast was injected at a rate of $3 \mathrm{ml} / \mathrm{s}$ in the present study. In Cohan et al. ${ }^{10}$ study the time to acquire corticomedullary phase images was not specified. They acquired corticomedullary phase images anywhere between 40-70 seconds. Nephrographic phase images were acquired after mean time of 163 seconds after initiation of contrast injection. In Szolar's ${ }^{11}$ study, corticomedullary phase images were acquired 50 seconds and 180 seconds after initiation of contrast injection respectively. So it is possible that the results in present study differed from results in Cohan et al. ${ }^{10}$ and Szolar et al. ${ }^{11}$ study.

Benign renal cysts.

Two radiologically benign cysts of Bosniak type II were diagnosed. The mean age in cases with benign cysts was $46 \pm$ 16.9 years (range - 34 to 58 years). Cysts demonstrated mean attenuation of $15 \pm 1.4 \mathrm{HU}$. The mean cyst enhancement was $1.5 \pm 3.5 \mathrm{HU}$ during corticomedulary phase and $2 \pm 2.8$
HU during nephrographic phase. The differences in mean enhancement in corticomedullary and nephrographic phases was statistically insignificant. These findings correlated with the study conducted by Birnbaum et al. ${ }^{5}$

In this series malignant renal mass was predominantly seen in older age group with higher male to female predominance. The malignant masses were predominantly ill-defined (8 0ut of 15 cases) whereas benign masses were predominantly well defined (12 out of 14 cases).

Similar results were seen in studies by Cohan et al. ${ }^{10}$, Birnbaum et al. ${ }^{5}$, Welch et al. ${ }^{7}$, Sun $\mathrm{M}$ et al. ${ }^{12}$, Seuong Won Choi et al. ${ }^{4}$

The malignant masses predominantly demonstrated heterogenous enhancement pattern in 13 out of 15 cases. A case of RCC measuring $2.1 \mathrm{~cm}$ displayed homogenous enhancement. A case of renal metastasis measuring about $2 \mathrm{~cm}$ from unknown primary also displayed homogenous enhancement

Similarly in a study by Kim et al. ${ }^{13}, \mathrm{RCC}$ above $3 \mathrm{~cm}$ in size predominantly demonstrated heterogenous enhancement. Homogenous enhancement was more commonly seen in RCC less than $3 \mathrm{~cm}$ in size.

In a study by Russo $\mathrm{P}$ et al. ${ }^{14}$ The enhancement pattern of a tumor is generally affected by its size; the larger a tumor grows $>5 \mathrm{~cm}$, the more frequently intratumoral necrosis or hemorrhage occurs.

In benign masses the homogenous and heterogenous enhancement pattern was equally distributed.

Furthermore in this study the attenuation value was useful in differentiating benign from malignant lesions. The mean attenuation value of malignant masses in unenhanced CT images was $34.8 \mathrm{HU}$ whereas in benign masses was 9.2 HU. In corticomedullary phase the malignant masses showed rapid enhancement with a mean $\mathrm{HU}$ value of $96.53 \pm$ 12.977 and a rapid decrease of in enhancement in following nephrographic phase with mean HU value of $72.93 \pm 10.194$. The differences in HU between corticomedullary phase and nephrographic phase in cases of malignant and benign masses was significant $(p<.05)$.

In our study 13 cases of RCC had a mean attenuation value of $34.31 \pm 2.2 \mathrm{HU}$ on a unenhanced scan. All cases of RCC showed significant contrast enhancement in corticomedullary phase $(99.54 \pm 9.12)$ and washout in nephrographic phase $(72 \pm 10.8)$.

The ROC curve analysis showed that the cut off values with highest sensitivity and specificity for characterization of RCC from other masses was $71.5 \mathrm{HU}$ in corticomedullary phase (sensitivity $100 \%$, specificity $99.9 \%$ ), $41.5 \mathrm{HU}$ in nephrographic phase (sensitivity 100\%, specificity 99.8\%).

\section{CONCLUSION}

In our study all renal masses were detected in both corticomedullary and nephrographic phases. For characterization of renal masses - the enhancement pattern, attenuation values in corticomedullary and nephrographic phases served as a valuable parameter in differentiating malignant from benign renal masses.

No statistically significant differences $(p>0.05)$ in enhancement were noted for the radiologically benign cysts 
when the corticomedullary and nephrographic phases were compared.

The study was primarily done to analyse attenuation values and enhancement pattern of renal masses during unenhanced, corticomedullary and nephrographic phases, for better detection and characterization of renal masses by multidetector computed tomography.

The malignant masses demonstrated greater enhancement in corticomedullary phase than in nephrographic phase (early enhancement - rapid washout)

The normal renal cortex demonstrated greater enhancement in nephrographic phase than in corticomedullary phase.

To conclude, MDCT protocol for evaluation of renal masses should include unenhanced, corticomedullary and nephrographic phases for better detection and characterization of renal masses.

\section{Limitations}

The threshold value we have reported is applicable only to patients with similar contrast injection protocols and scan delay times because the enhancement pattern could vary according to contrast injection variables and scan delay times. This study did not evaluate differences of each subtype of $\mathrm{RCC}$ because of small sample size.

\section{REFERENCES}

1. Goldman LW. Principles of CT: multislice CT. J Nucl Med Technol. 2008;36(2):57-68-76.

2. Eble JN, Sauter G, Epstein JI SI. Pathology and genetics of tumours of the urinary system and male genital organs. IARC Press Lyon, France. 2004;

3. Bosniak MA. The current radiological approach to renal cysts. Radiology. 1986;158(1):1-10.

4. Choi S, Jeon SH, Chang S. Urological Oncology Characterization of Small Renal Masses Less than $4 \mathrm{~cm}$ with Quadriphasic Multidetector Helical Computed Tomography: Differentiation of Benign and Malignant Lesions. Korean J Urol. 2012;53(3):159-64.

5. Birnbaum BA, Jacobs JE, Ramchandani P. Multiphasic renal CT: comparison of renal mass enhancement during the corticomedullary and nephrographic phases. Radiology. 1996;200(3):753-8.

6. Shetty C, Lakhar B, Devi B, Lakshmi B. Dual-phase helical CT of kidney : Comparison of corticomedullary and nephrographic phases in detection and characterization of renal masses. Indian J Radiol Imaging [Internet]. 2004;14(3):285-90.

7. Welch TJ, LeRoy AJ. Helical and electron beam CT scanning in the evaluation of renal vein involvement in patients with renal cell carcinoma. J Comput Assist Tomogr. 1997;21(3):467-71.

8. Weyman PJ, McClennan BL, Lee JK, Stanley RJ. CT of calcified renal masses. AJR Am J Roentgenol. 1982;138(6):1095-9.

9. Israel GM, Bosniak MA. Calcification in cystic renal masses: is it important in diagnosis? Radiology. 2003;226(1):47-52.

10. Cohan RH, LS S, M K, JC B, IR F. Renal masses: assessment of corti- comedullary-phase and nephrographic-phase CT scans. Radiology. 1995;96:445-51.
11. Szolar D, Kammerhuber F, Aliziebler S, Al E. Multiphasic helical CT of the kidney: increased conspicuity for detection and characterization of small (_3 cm)renal masses. Radiology. 1997;202:211-7.

12. Sun M, Abdollah F, Bianchi M, Trinh Q-D, Jeldres C, Tian Z, et al. A stage-for-stage and grade-for-grade analysis of cancer-specific mortality rates in renal cell carcinoma according to age: a competing-risks regression analysis. Eur Urol. 2011;60(6):1152-9.

13. Park H, Park JY, Kim DY, Ahn SH, Chon CY, Han $\mathrm{KH}$, et al. Characterization of focal liver masses using acoustic radiation force impulse elastography. World J Gastroenterol. 2013;19(2):219-26.

14. Russo P. Renal cell carcinoma: presentation, staging, and surgical treatment. Semin Oncol. 2000;27(2):160-76.

Source of Support: Nil; Conflict of Interest: None

Submitted: 20-05-2018; Accepted: 21-06-2018; Published online: 07-07-2018 